\title{
A neutrophil elastase inhibitor prevents bleomycin-induced pulmonary fibrosis
} in mice

\author{
Akihiro Takemasa, Yoshiki Ishii and Takeshi Fukuda
}

ABSTRACT: Neutrophil elastase plays pivotal roles in the pathogenesis of pulmonary fibrosis. The neutrophil elastase inhibitor, sivelestat, could alleviate pulmonary fibrosis; however, the antifibrotic mechanisms have not yet been clarified. We examined the antifibrotic mechanisms, mainly focusing on a key fibrotic cytokine, transforming growth factor (TGF)- $\beta 1$, in this study.

To elucidate the antifibrotic mechanisms of sivelestat, we examined a murine model of bleomycin-induced early-stage pulmonary fibrosis. After intratracheal instillation of bleomycin, sivelestat was administered intraperitoneally once a day for 7 or 14 days. Bronchoalveolar lavage fluid and lung samples were examined on day 7 or day 14 after bleomycin instillation.

In the bleomycin-induced early-stage pulmonary fibrosis model, the neutrophil elastase level was increased in the lungs. Sivelestat significantly inhibited the increase in lung collagen content, fibrotic changes, the numbers of total cells (including macrophages, neutrophils and lymphocytes), the levels of the active form of TGF- $\beta 1$ and phospho-Smad2 in bleomycin-induced earlystage pulmonary fibrosis. The total TGF- $\beta 1$ levels and relative changes of TGF- $\beta 1$ mRNA expression, however, were not decreased significantly by sivelestat.

These results suggest that sivelestat alleviated bleomycin-induced pulmonary fibrosis via inhibition of both TGF- $\beta$ activation and inflammatory cell recruitment in the lung.

KEYWORDS: Early-stage pulmonary fibrosis, neutrophil elastase, sivelestat, transforming growth factor- $\beta 1$

$\mathbf{P}$ ulmonary fibrosis is caused by a variety of insults to the lung, such as acute lung injury, irradiation and drugs [1]. The degree of fibrosis is a major determinant of clinical outcome in patients with fibrotic pulmonary diseases because current therapies are ineffective or only marginally effective.

Acute lung injury (ALI) or acute respiratory distress syndrome (ARDS) is associated with acute and diffuse alveolar damage, noncardiogenic pulmonary oedema and subsequent pulmonary fibrosis. Based on a recent report [2], the mortality of ARDS was $40-45 \%$ among patients in observational studies and $35-40 \%$ among those in randomised controlled trials. An important issue for the long-term survival of patients with ARDS is the degree of parenchymal fibrosis and loss of pulmonary function [1].

The fibroproliferative phase of ALI/ARDS has traditionally been regarded as a late event.
However, recent studies suggest that increased collagen turnover is an early event, occurring within $24 \mathrm{~h}$ of diagnosis [3]. Extensive thin-section computed tomographic abnormalities, including traction bronchiolectasis, suggest that fibroproliferative changes were independently predictive of poor prognosis in ARDS patients within 7 days of onset [4]. Transforming growth factor (TGF)- $\beta$, especially its isoform TGF- $\beta 1$, is a profibrotic cytokine that is overexpressed in patients with fibrosis and was directly associated with fibrosis in multiple human settings and animal models [5]. Elevated levels of active TGF- $\beta 1$ were documented not only in bronchoalveolar lavage fluid (BALF) but also in tissue samples within $24 \mathrm{~h}$ after the diagnosis of ARDS [6].

The presence of fibrotic changes early in the course of ARDS has been associated with an increased risk of mortality [3, 4, 7]. Fibroproliferation in the early response to lung injury could be an important therapeutic target. Idiopathic pulmonary fibrosis

\section{AFFILIATIONS}

Dept of Pulmonary Medicine and Clinical Immunology, Dokkyo Medical University School of Medicine, Tochigi, Japan.

\section{CORRESPONDENCE}

Y. Ishii

Dept of Pulmonary Medicine and Clinical Immunology

Dokkyo Medical University School of Medicine

800 Kitakobayashi

Mibu

Tochigi

321-0293

Japan

E-mail: ishiiysk@dokkyomed.ac.jp

Received:

July 252011

Accepted after revision:

Feb 252012

First published online:

March 222012 
(IPF) is also a progressive and fatal fibrotic pulmonary disease without effective available therapies.

Bleomycin (BLM)-induced inflammation and fibrosis represent an experimental model for IPF as well as ALI/ARDS. Recent studies have suggested that increased collagen turnover and fibrotic changes are earlier events within 14 days in the BLMinduced fibrosis [8-11] and these changes were used commonly as a pulmonary fibrosis model. Signs of fibrosis were observed at 7 days after BLM administration [8]. A significant increase in total collagen deposition was observed as early as day 8 , which continued to increase until day 16 [9]. Hence, we used this BLM-induced model as an early-stage fibrosis model within 14 days.

Neutrophil elastase (NE) is a serine proteinase that is released from activated neutrophils and has broad substrate specificity. NE has been presumed to play a role in degenerative and inflammatory diseases by its proteolysis of the extracellular matrix [12, 13], and contribute to lung injury [14]. NE induces lung epithelial apoptosis via proteinase-activated receptor (PAR)-1 [14], and PAR-1 could play an important role in inflammatory and fibroproliferative responses in ALI/ARDS $[10,15]$. The serum NE level was elevated in patients with pulmonary fibrosis $[16,17]$, and some evidence does suggest that inhibition of its activity could attenuate the development of the disease [13, 18, 19].

In this study, we examined the antifibrotic effects of a specific NE inhibitor, sivelestat, mainly on TGF- $\beta 1$ activation in the BLM-induced early-stage pulmonary fibrosis model. We found that sivelestat alleviated BLM-induced pulmonary fibrosis via inhibition of TGF- $\beta 1$ activation and inflammatory cell recruitment in the lungs. Our results suggest that we may be able to alleviate not only inflammatory but also fibroproliferative processes in IPF as well as ALI/ARDS.

\section{METHODS}

See the online supplementary material for details on methods.

\section{Animal treatment}

Male C57BL/6 mice (8-10 weeks of age; Japan Clea, Tokyo, Japan) were allocated to one of four groups: 1) intratracheal saline plus saline intraperitoneally (control group); 2) intratracheal BLM plus saline i.p. (BLM group); 3) intratracheal BLM plus $100 \mathrm{mg} \cdot \mathrm{kg}^{-1}$ of sivelestat (Ono Pharmaceuticals, Osaka, Japan) in saline i.p. (BLM+sivelestat group); and 4) intratracheal saline plus $100 \mathrm{mg} \cdot \mathrm{kg}^{-1}$ of sivelestat in saline i.p. (sivelestat group). BLM (5 mg. $\mathrm{kg}^{-1}$; Nippon Kayaku, Tokyo, Japan) in $75 \mu \mathrm{L}$ saline or saline alone was intratracheally administered to the mice on day 0 . Sivelestat or saline was given i.p. $3 \mathrm{~h}$ after BLM instillation on day 0 , and then once a day for 7-14 days. Immediately after sacrifice on day 7 and day 14, bronchoalveolar lavage was performed and the lungs were removed en bloc. Some lung samples were used for histopathological examination, collagen assay and quantitative real-time PCR. The rest were used as homogenate samples with sonication. All experiments were performed in accordance with the National Institutes of Health guidelines. The protocols were approved by the Dokkyo Medical University School of Medicine Subcommittee on Research Animal Care (Tochigi, Japan).

\section{Bronchoalveolar lavage and homogenate lung samples}

After counting the cell numbers in the BALF, cells were cytospun and stained with Diff-Quick for cell classification. The homogenised lung samples and the BALF supernatant were freeze-dried using a lyophiliser. The lyophilised samples were dissolved in saline for determination of NE activity, the active form of TGF- $\beta 1$, total TGF- $\beta 1$ and phospho-Smad2.

\section{NE activity}

NE activity was determined using the NE-specific substrate $N$ methoxysuccinyl-Ala-Ala-Pro-Val p-nitroanilide (Calbiochem, San Diego, CA, USA). Fluorescence was measured at wavelengths of $360 \mathrm{~nm}$ for excitation and $460 \mathrm{~nm}$ for emission.

\section{Quantitative determination of active and total TGF- $\beta 1$}

The concentrations of murine active and total TGF- $\beta 1$ were determined using ELISA kits (R\&D Systems, Minneapolis, MN, USA).

\section{Histopathological evaluation}

The right lung was fixed in 10\% buffered formalin, and stained with hematoxylin-eosin and Masson's trichrome. Histopathological grading of fibrosis was performed by three experienced histopathologists using a blinded semiquantitative scoring system [20].

\section{Collagen assay}

The collagen content in the homogenate of left lung sample was determined using the Sircol Collagen Assay kit (Biocolor Ltd, Belfast, UK) [20].

\section{Real-time PCR analysis}

Quantification of TGF- $\beta 1$ mRNA expression was performed by real-time PCR on an ABI Prism 7700 sequence detection system (PE Applied Biosystems, Foster City, CA, USA), by using SYBR green (Roche Diagnostics, Somerville, MA, USA) as a doublestranded DNA-specific binding dye. Glyceraldehyde-3-phosphate dehydrogenase was used as an internal control.

\section{Phospho-Smad2 measurement}

Western blot and densitometric analysis were performed as usual with phospho-Smad2 (Ser465/467) (Cell Signaling Technologies, Danvers, MA, USA) and actin antibodies (sc8432; Santa Cruz Biotechnology, Santa Cruz, CA, USA). The Sandwich ELISA PathScan ${ }_{\circledast}$ Phospho-Smad2 (Ser465/467) Kit (Cell Signaling Technologies) was used to measure the concentration of phospho-Smad2.

\section{Statistical analysis}

Data are expressed as mean \pm SEM. Statistical significance was determined by one-way ANOVA or paired t-test. p-values $<0.05$ were considered significant.

\section{RESULTS}

\section{BLM-induced pulmonary fibrosis}

Histopathological examination revealed that BLM induced marked inflammatory cell infiltration with fibrosis in the lungs on day 14 (fig. 1c, g and i). In BLM-treated mice, sivelestat prevented the BLM-induced pulmonary fibrosis significantly (fig. $1 \mathrm{~d}, \mathrm{~h}$ and $\mathrm{i}$ ). The collagen assay demonstrated that sivelestat reduced the production of total collagen in BLMtreated lungs (fig. 1j). Sivelestat in non-BLM-treated mice 

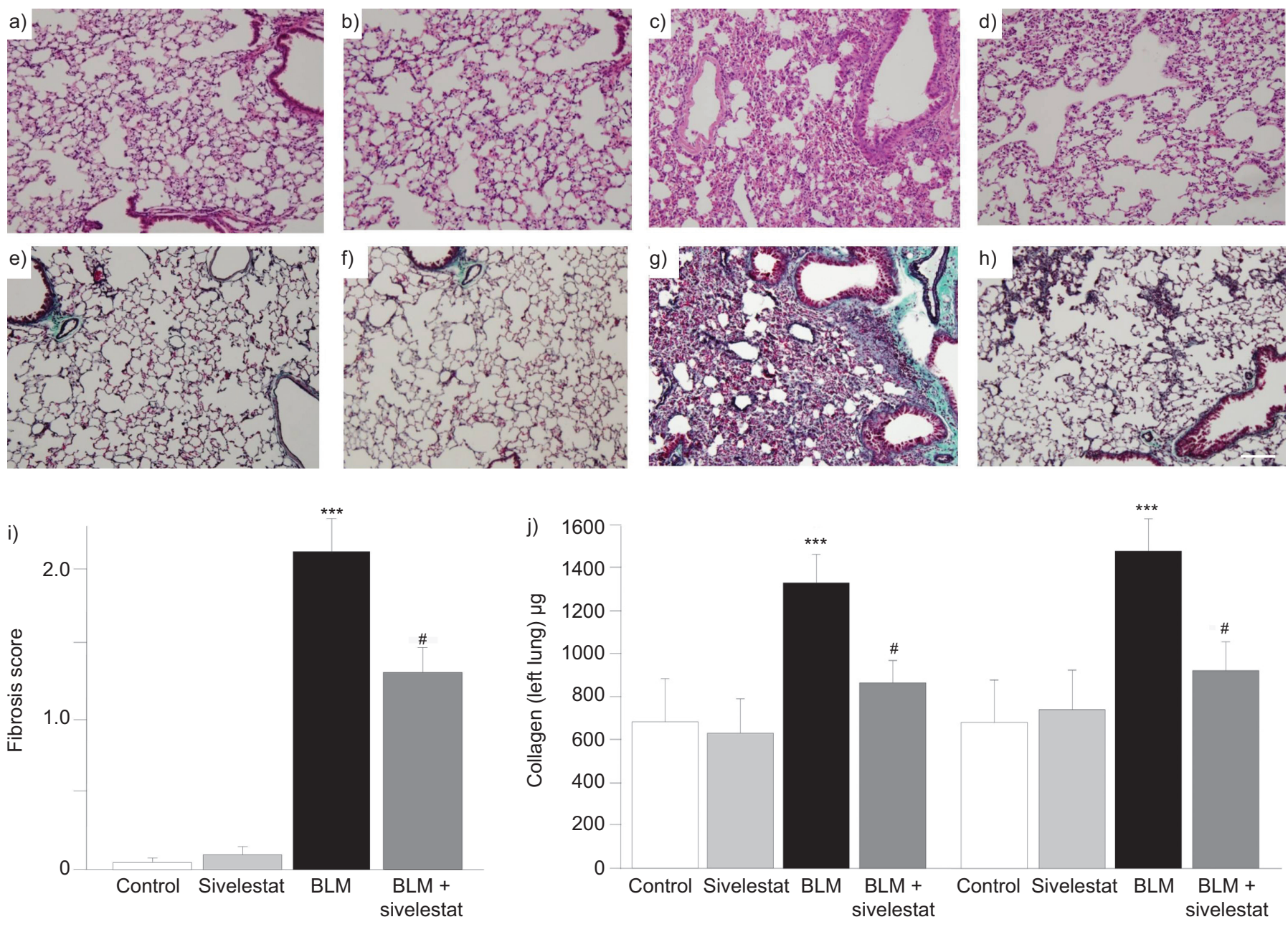

$* * *$

Day 7

FIGURE 1. The antifibrotic effects of sivelestat were observed by histological examination. Representative pathological findings in lung tissue stained with a-d) hematoxylin-eosin and e-h) Masson's trichrome on day 14 after bleomycin (BLM) instillation. a and e) Control group; b and f) sivelestat group; c and g) BLM group; d and h) BLM+sivelestat group. Sivelestat $\left(100 \mathrm{mg} \cdot \mathrm{kg}^{-1}\right)$ was given $3 \mathrm{~h}$ after intratracheal BLM instillation $\left(5 \mathrm{mg} \cdot \mathrm{kg}^{-1}\right)$ and daily from day 2 to 14 after intratracheal BLM instillation. i) Fibrosis scoring based on severity and area of fibrosis on day 14 . The fibrosis score of mice treated with sivelestat $100 \mathrm{mg} \cdot \mathrm{kg}^{-1}$ without BLM showed no significant change compared with that of the untreated control mice. Sivelestat $100 \mathrm{mg} \cdot \mathrm{kg}^{-1}$ significantly alleviated BLM-induced pulmonary fibrosis. j) Collagen content in lung tissues. BLM augmented the lung collagen content on day 7 as well as day 14 . Sivelestat $100 \mathrm{mg} \cdot \mathrm{kg}^{-1}$ significantly alleviated the increased collagen content on day 7 as well as day 14 . Data are presented as mean \pm SEM; $n=10$ in each group. Scale bar $=200 \mu \mathrm{m}$. ${ }^{* \star}: \mathrm{p}<0.001$ versus control group; ${ }^{\#}: \mathrm{p}<0.001$ versus BLM group.

generated no change in lung morphology (fig. $1 \mathrm{~b}$ and $\mathrm{f}$ ) and the pathological fibrosis score (fig. 1i).

\section{Cell analysis of BALF}

The cell counts and cell differentiation in BALF were examined to study sivelestat effects on the BLM-induced inflammatory responses. BLM elevated the number of inflammatory cells, including the cell counts of total cells, macrophages, neutrophils and lymphocytes, on days 7 and 14 (fig. 2a-d). Sivelestat significantly reduced inflammatory cell recruitment in the lungs on days 7 and 14 (fig. 2a-d). Sivelestat alone generated no change in the number of inflammatory cells (fig. 2a-d).

\section{NE activity}

In the BLM group, NE activity was elevated in BALF and lung homogenate samples on days 7 and 14 (fig. 3a and b). Daily sivelestat administration significantly reduced NE activity on days 7 and 14 (fig. 3a and b). NE activity in lung homogenate samples showed similar results as those in BALF on days 7 and 14 (fig. 3a and b). The sivelestat group showed that sivelestat alone generated no change of NE activity in comparison with the control group (data not shown).

\section{Quantitative analysis of the active form of TGF- $\beta 1$ and total TGF- $\beta 1$}

The active form of TGF- $\beta 1$ and total TGF- $\beta 1$ increased from day 7 to day 14 in the BLM group, compared with the control group (fig. $4 \mathrm{a}$ and $\mathrm{b}$ ). Total TGF- $\beta 1$ increased from day 7 to day 14 in the BLM+sivelestat group, similarly to the BLM group (fig. $4 \mathrm{a}$ and $\mathrm{b}$ ). In contrast with the BLM group, the active form of TGF- $\beta 1$ did not increase from day 7 to day 14 in the BLM+sivelestat group (fig. $4 \mathrm{a}$ and $\mathrm{b}$ ). The results of lung homogenate samples were similar to those of the BALF samples (fig. 4c and d). Sivelestat alone generated no change 

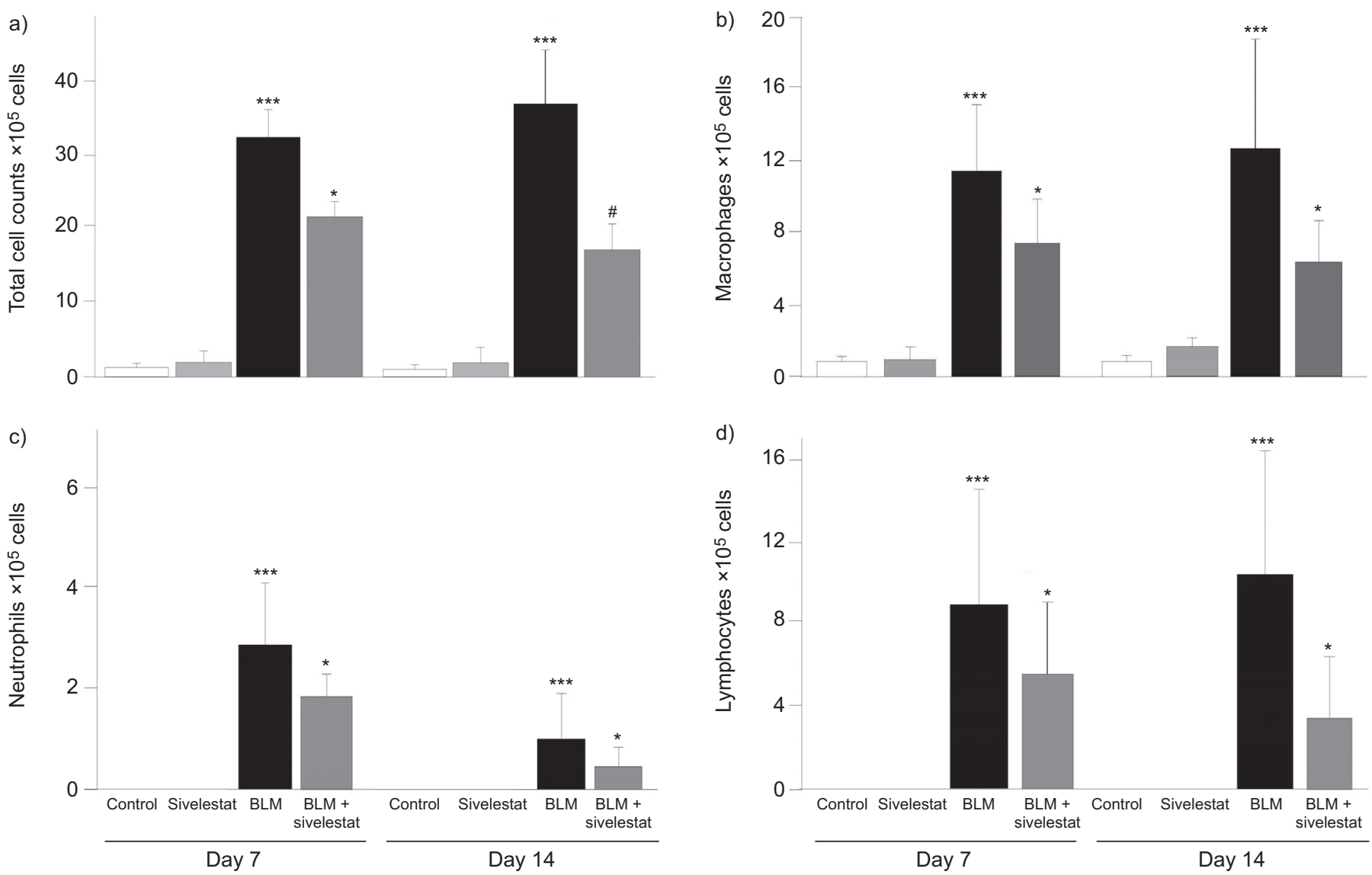

FIGURE 2. Sivelestat reduced the numbers of inflammatory cells in bronchoalveolar lavage fluid. The effect of sivelestat on the results of bronchoalveolar lavage analysis. a) Total cell counts on day 7 and day 14; b) macrophage cell counts on day 7 and day 14 ; c) neutrophil cell counts on day 7 and day 14 ; d) lymphocyte cell counts on day 7 and day 14 . Data are presented as mean \pm SEM. $n=10$ in each group. BLM: bleomycin. ${ }^{*}: p<0.05$ versus BLM group; ***: $p<0.001$ versus control group; ${ }^{*}: p<0.001$ versus BLM group.

of the active TGF- $\beta 1$ and total TGF- $\beta 1$ in comparison with the control group (data not shown).

\section{Expression of TGF- $\beta 1$ mRNA}

The relative TGF- $\beta 1$ mRNA expression was significantly higher in the BLM group than that in the control group on day 7 and 14 (fig. 5). In the BLM+sivelestat group, TGF- $\beta 1$ mRNA expression was as high as that in the BLM group (fig. 5). This finding indicated that sivelestat did not suppress the increased expression of TGF- $\beta 1$ mRNA induced by BLM.

\section{Phospho-Smad2 measurement}

Phospho-Smad2 is a downstream signalling molecule of TGF$\beta 1$, and phosphorylation of Smad2 is regarded as a crucial intracellular step in TGF- $\beta 1$ signal transduction [21]. The phospho-Smad2 protein levels determined by Western blotting were significantly increased in the BLM group compared with the control group (fig. 6a). Sivelestat treatment demonstrated a significant decrease of the phospho-Smad2 protein level induced by BLM. Sivelestat alone generated no change of Smad2 phosphorylation in comparison with the control group (data not shown). ELISA for phospho-Smad2 indicated similar results to those of the Western blot (fig. 6b).

\section{DISCUSSION}

$\mathrm{NE}$ is an elastolytic enzyme with a broad substrate range that can digest most proteins. It has been thought to play a role in degenerative and inflammatory diseases [12, 13]. Being involved in inflammatory lung injury [1], apoptosis [14] and fibroproliferative responses via PAR-1 signal transduction [10, $14,15]$, NE could contribute to the pathogenesis of various pulmonary diseases.

In the present study, to elucidate the possible involvement of $\mathrm{NE}$ in pulmonary fibrosis, we investigated a specific NE inhibitor, sivelestat, in BLM-induced early-stage pulmonary fibrosis. Sivelestat significantly inhibited BLM-induced fibrotic changes as well as inflammatory cell accumulation in the lungs.

It has long been recognised that NE activity in serum and BALF is significantly elevated in patients with pulmonary fibrosis such as IPF, and NE could play an important role in the process of pulmonary fibrosis [16, 17]. The paradoxical mechanisms by which NE breaks down the extracellular matrix, whereby it can promote excess matrix deposition, have not yet been fully defined. Pulmonary fibrosis is the central pathological endpoint of various causes, such as progressive inflammation, cell death and disordered repair [1]. It has been hypothesised that 

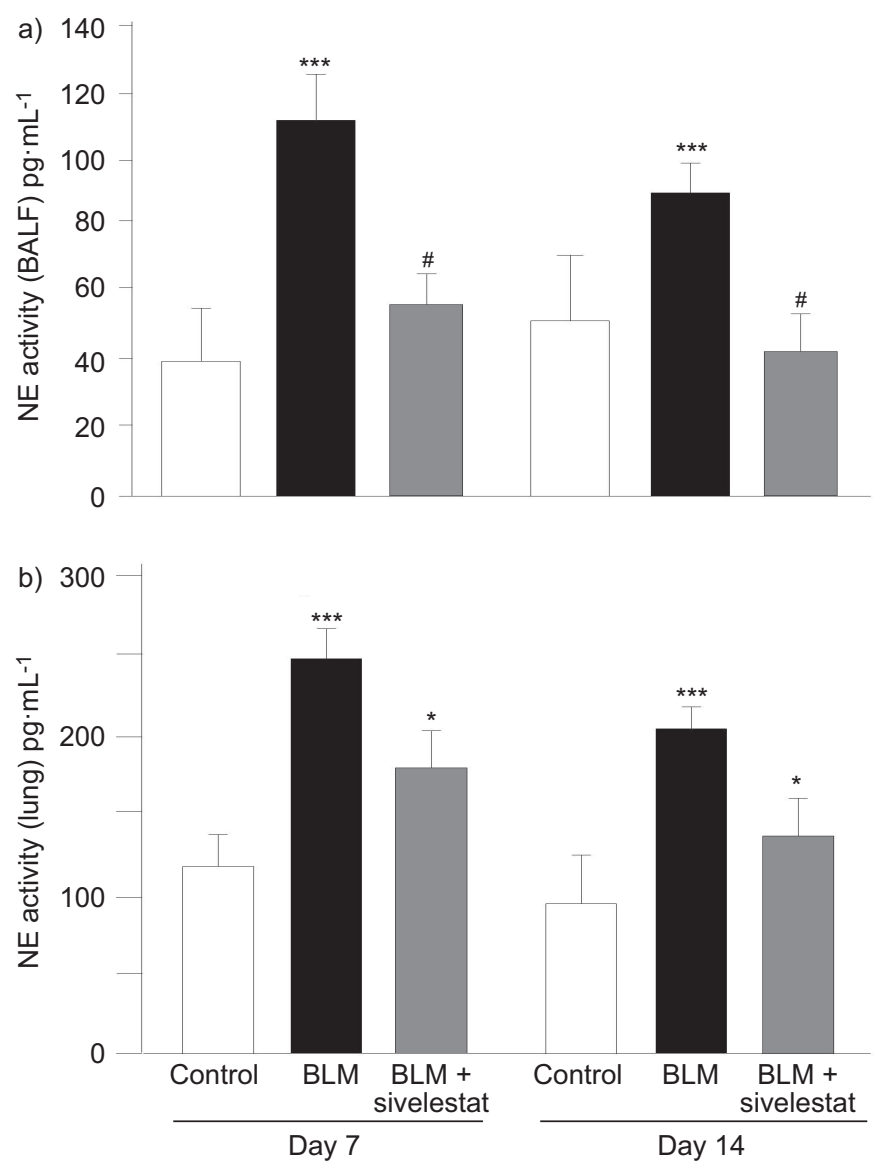

FIGURE 3. Sivelestat significantly inhibited neutrophil elastase (NE) activity in bronchoalveolar lavage fluid (BALF) as well as in lung homogenate samples. a) NE activity in BALF on day 7 and day 14; b) NE activity in lung homogenate samples on day 7 and day 14. Data are presented as mean \pm SEM. $n=10$ in each group. BLM: bleomycin. *: $p<0.05$ versus BLM group; ***: $p<0.001$ versus control group; * $\mathrm{p}<0.001$ versus BLM group.

fibrotic changes are the result of successive inflammation and injuries to the alveolar membrane with ineffective reconstitution of normal epithelium [22]. The core of this hypothesis is that alveolar epithelial cells fail to repair the damaged epithelium as a result of excessive tissue repair which leads to interstitial fibrotic changes and scarring. Based on this hypothesis, inhibition of lung injury could alleviate pulmonary fibrosis.

In the present study, sivelestat inhibited inflammatory cell recruitment in the lungs, which is consistent with previous reports [13]. It was reported that NE can enhance neutrophil accumulation. NE enhances neutrophil-dependent inflammation by eliminating the local anti-inflammatory activity of progranulin [23]. NE also stimulates bronchial epithelial cells and macrophages to produce neutrophil chemoattractants such as interleukin (IL)-8 [12]. Therefore, inhibition of NE activity by sivelestat could attenuate neutrophil accumulation.

Several previous studies reported that inhibition of NE activity could attenuate the development of pulmonary fibrosis $[13,18$, 19], and sivelestat could alleviate fibrotic changes [13]. A hypothesis on the mechanism of the suppressive effect of sivelestat on fibrosis development has largely centred on responses to injury and inflammatory reaction. Animal experiments showed that the suppressive effect of sivelestat was observed when the drug was administered during the acute stage within 14 days of onset of fibrosis [13]. It might therefore be possible for sivelestat to attenuate fibrosis during the neutrophil-dominant acute stage of pulmonary fibrosis.

In the present study, we demonstrated that sivelestat inhibited not only inflammatory cell recruitment, but also TGF- $\beta 1$ activation in the lungs. Based on the fact that marked neutrophilic alveolitis was observed and fibrotic changes were attenuated in BLM-treated NE-deficient mice, CHUA et al. [19] concluded that NE could induce TGF- $\beta$ activation, and NE-deficient mice showed diminished pulmonary fibrosis through impairment of TGF- $\beta$ activation. In the present study, we found that sivelestat significantly reduced the levels of the active form of TGF- $\beta 1$ in BALF and lung homogenates, but not the total TGF- $\beta$ levels. Sivelestat did not affect the BLM-induced increase in TGF- $\beta 1$ mRNA expression. Moreover, phosphorylation of Smad2 was significantly reduced in the BLM+sivelestat group. These findings indicate that sivelestat inhibited NE-mediated activation of TGF- $\beta 1$ and subsequent lung fibrosis. This is a newly found mechanism through which sivelestat inhibits NE-mediated fibrosis, and supports a previous report using NE-/- mice [19].

Although some factors could work through a TGF- $\beta$-independent pathway [21], and intricately intertwined mechanisms are associated with fibrosis, evidence for the eminence of TGF- $\beta 1$ in fibrosis is substantial. In fibrogenesis, TGF- $\beta$ induces myofibroblast differentiation and extracellular matrix synthesis, and inhibits matrix degradation [24]. TGF- $\beta$ exists in three isoforms, TGF- $\beta 1$, TGF- $\beta 2$ and TGF- $\beta 3$. The isoforms differ in their binding affinity for TGF- $\beta$ receptors, and TGF- $\beta 1$ is generated in greatest abundance subsequent to tissue damage. Previous studies have shown that BLM induces collagen deposition and that TGF- $\beta 1$ plays an important role in mediating this process [25].

TGF- $\beta 1$ is produced by a variety of cells, including epithelial cells, alveolar macrophages, fibroblasts and endothelial cells [26]. Alveolar macrophages are thought to be one of major sources of TGF- $\beta 1$, because it has been reported that macrophages release large amounts of TGF- $\beta 1$ in IPF patients and that TGF- $\beta 1$ is present in fibroblastic foci in IPF patients' biopsies [24]. TGF- $\beta$ is most efficiently secreted as a large latent complex and most TGF- $\beta$ is stored in the extracellular matrix before activation [19]. A key feature of IPF is the presence of high levels of inactive precursor form TGF- $\beta 1$ in the lung parenchyma [27]. The main sources could be the accumulated TGF- $\beta 1$ in the lung parenchyma, especially in the initial fibrotic changes. Therefore, elevated levels of TGF- $\beta 1$ as well as TGF- $\beta$-inducible proteins, such as procollagen type III, have been demonstrated in the lungs of patients with ARDS as early as $24 \mathrm{~h}$ after onset $[6,7]$.

The latent form of TGF- $\beta 1$ can be activated by a number of stimuli $[6,19]$, including proteases such as plasmin, metalloproteinases, radiation, reactive oxygen species (ROS), thrombospondin- 1 , integrin $\alpha v \beta 6$, CD44 and IL-13. CHUA et al. [19] proposed the concept that NE can support matrix production and tissue repair via TGF- $\beta$ activation. Current evidence suggests that activation of proteinase-activated receptors (PARs), a subfamily of the seven transmembrane domain G-protein-coupled receptors, may be of particular importance in influencing inflammatory and 

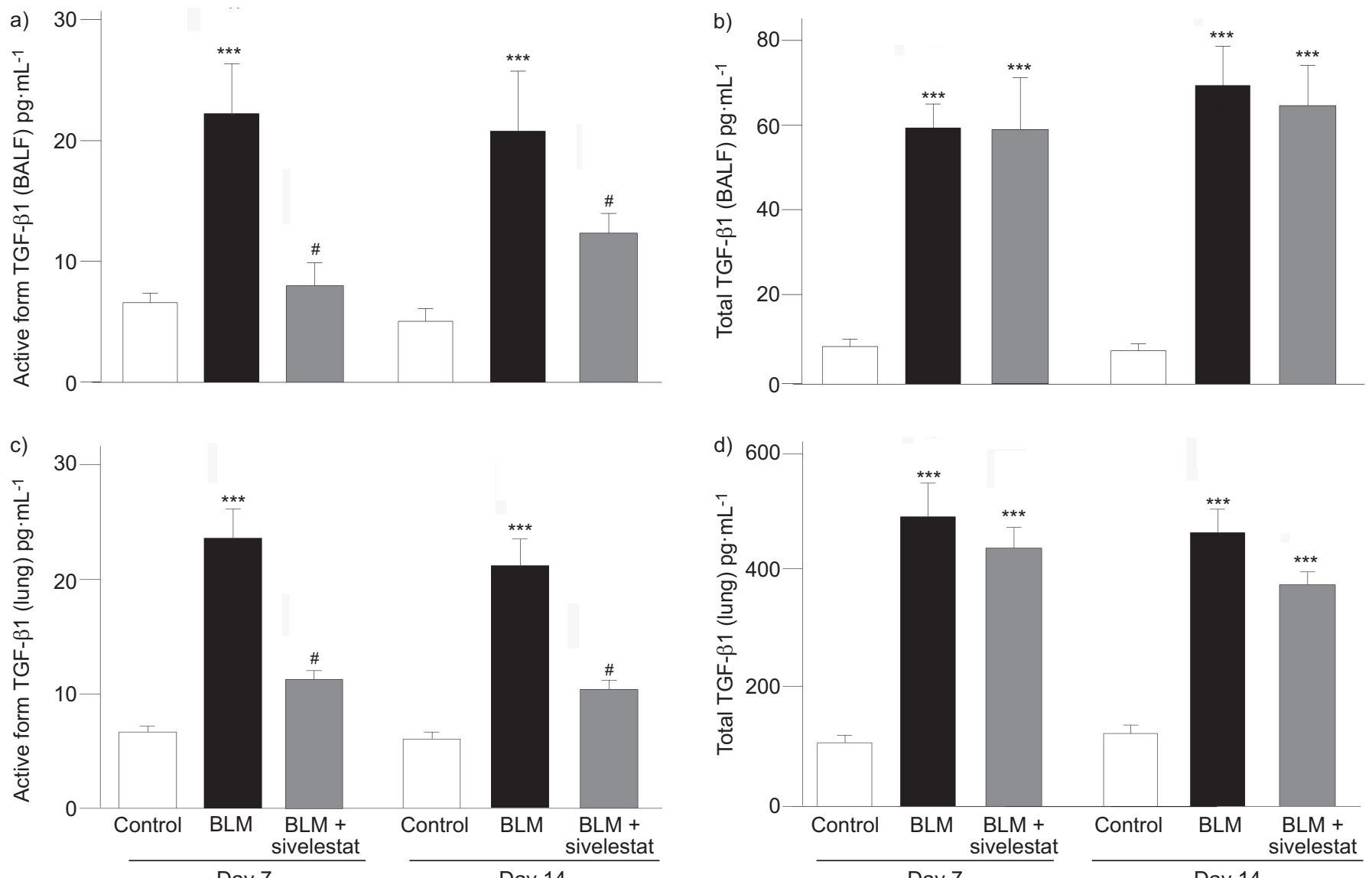

FIGURE 4. Sivelestat significantly inhibited the formation of the active form of transcription growth factor (TGF)- $\beta 1$, but not total TGF- $\beta 1$ in bronchoalveolar lavage fluid (BALF) as well as in lung homogenate samples. a) The active form of TGF- $\beta 1$ in BALF on day 7 and day 14 ; b) total TGF- $\beta 1$ in BALF on day 7 and day 14 ; c) active form of TGF- $\beta 1$ in lung homogenate samples on day 7 and day 14 ; d) total TGF- $\beta 1$ in lung homogenate samples on day 7 and day 14 . Data are shown as mean \pm SEM. $n=10$ in each group. BLM: bleomycin. ${ }^{* *}: p<0.001$ versus control group; ${ }^{*}: p<0.001$ versus BLM group.

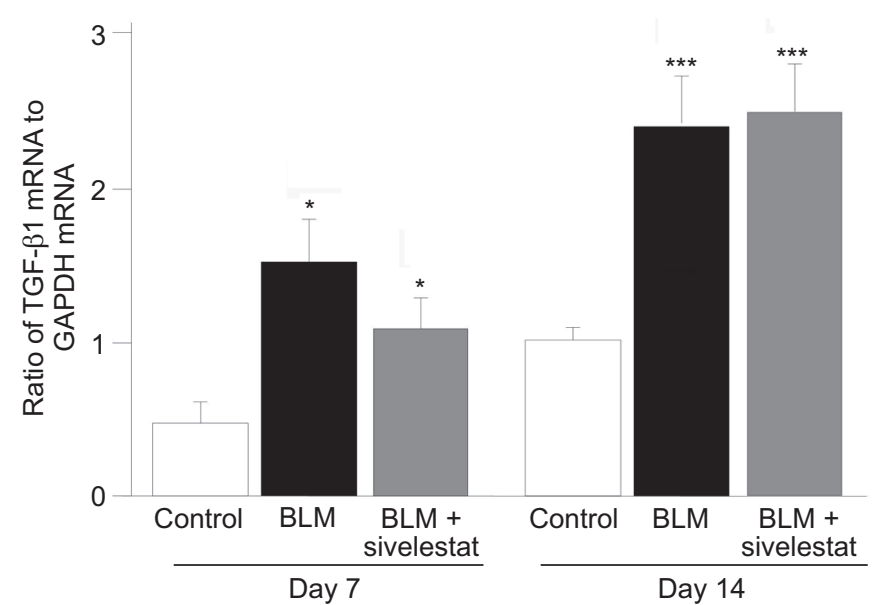

FIGURE 5. Sivelestat did not inhibit expression of transcription growth factor (TGF)- $\beta 1$ mRNA in the lungs with bleomycin (BLM)-induced pulmonary fibrosis. The ratio of TGF- $\beta 1$ mRNA expression to glyceraldehydes-3-phosphate dehydrogenase (GAPDH) mRNA expression we analysed using real-time reverse transcriptase PCR in lung homogenate samples, on days 7 and 14. Data are presented as mean \pm SEM. $n=10$ in each group. *: $p<0.05$ versus control group, $* * *: p<0.001$ versus control group. fibroproliferative responses in ALI/ARDS. The PAR family is comprised of four members (PAR1-PAR4) and perpetuates the interplay between coagulation, inflammation and fibrotic changes [15]. SCOTTON et al. [10] reported that PAR1-dependent signalling has the capacity to drive fibrotic responses by activating TGF- $\beta$ and influencing fibroblast-to-myofibroblast differentiation. Since NE can activate PAR1 [14], the elevated level of NE in patients with ARDS could activate TGF- $\beta$ through the PAR.

Although the precise mechanism remains to be elucidated and further examination would be preferable, there are theories on the mechanism of TGF- $\beta 1$ activation by NE, which include a direct activation mechanism, indirect ones through the PARs and other unknown pathways.

While inhibiting TGF- $\beta 1$ activation is not always effective in treating pulmonary fibrosis, sivelestat could alleviate not only inflammation but also TGF- $\beta 1$-dependent fibrosis in the early stage where excessive NE activity might be involved. In response to inflammatory stimuli, neutrophils migrate to inflammatory tissues, where they efficiently bind, engulf and inactivate pathogenic agents. This is known as inflammation in association with neutrophil sequestration in the pulmonary microvasculature. Neutrophils sequester the lesions and deliver a high local concentration of proteolytic enzymes and 
a)
$60 \mathrm{kDa}$

$43 \mathrm{kDa}$
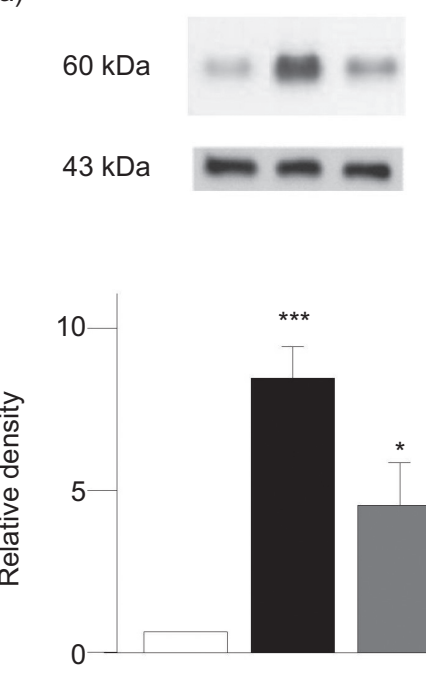

Day 7

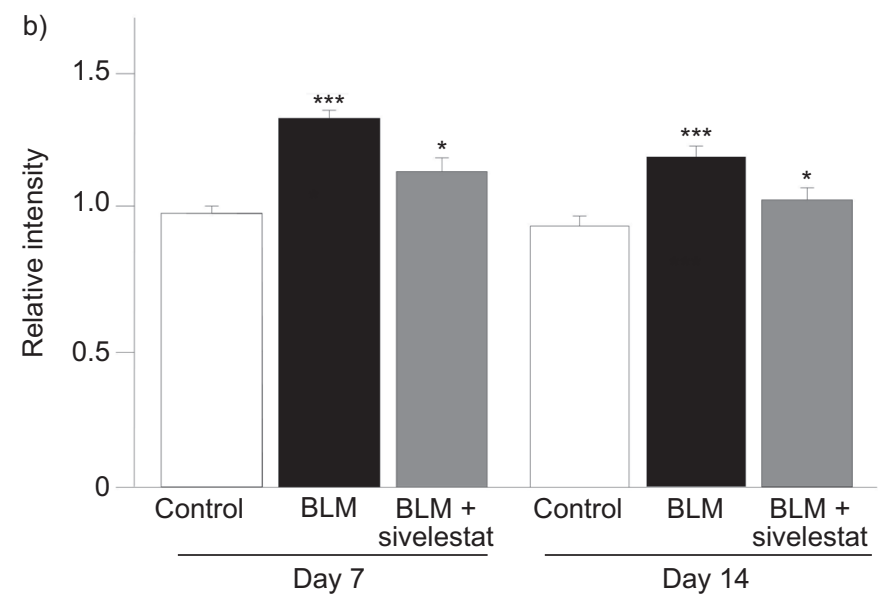

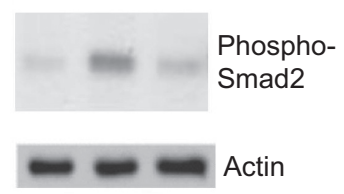

Phospho-

Actin
FIGURE 6. Sivelestat significantly inhibited Smad2 phosphorylation in bleomycin (BLM)-induced pulmonary fibrosis. a) Representative figure of phosphoSmad2 protein and quantitation to actin protein by Western blot analysis in lung homogenates on days 7 and 14; b) sandwich ELISA analysis of phospho-Smad2 protein in lung homogenates on days 7 and 14 . Data are presented as mean \pm SEM $\mathrm{n}=10$ in each group. *: $\mathrm{p}<0.05$ versus BLM group; ${ }^{* *}: \mathrm{p}<0.001$ versus control group.

ROS [28]. It is regulated by many endogenous protease inhibitors, such as $\alpha_{1}$-antitrypsin with a molecular weight of $\sim 52 \mathrm{kDa}$. However, these macromolecular protease inhibitors have difficulty passing through the sequestered interstitial space of pulmonary tissue. Alternatively, the inhibitory function can be performed by the low molecular weight $(528.51 \mathrm{Da})$ sivelestat [12]. In contrast to endogenous protease inhibitors, sivelestat is structurally resistant to ROS inactivation and is effective in inhibiting tissue-bound NE [12]. In addition, sivelestat could alleviate not only inflammatory reactions but also TGF- $\beta 1$ activation leading to pulmonary fibrosis.

Since sivelestat could alleviate the NE-induced lung epithelial apoptosis [14], aerosolised administration of the drug could benefit patients. However, aerosolised sivelestat may not be sufficient to abrogate NE-induced pulmonary fibrosis, because, being delivered only through airways, it may not adequately reach the lung parenchyma containing high levels of TGF- $\beta 1$. Dual-route administration of sivelestat intratracheally and intravenously could offer additive or synergistic effects compared with the single venous pathway. Hence, intratracheal administration is an attractive strategy, requiring further investigation.

In summary, our results demonstrate that inhibition of NE by sivelestat suppresses both neutrophilic inflammation and TGF- $\beta$ activation, which resulted in the suppression of BLM-induced pulmonary fibrosis. These data may lead to new therapeutic approaches in ARDS through more targeted inhibition of fibrosis. A key point highlighted is that fibroproliferation in ARDS occurs with the summation of deeply intertwined processes, and it is likely to begin immediately after the onset of injury. Sivelestat exerted its effect in the early fibrotic stage, in which excessive NE activity could be involved. In this stage, sivelestat could alleviate not only inflammatory reaction but also TGF- $\beta 1$ activation leading to pulmonary fibrosis. If sivelestat is administered as early as possible, we could alleviate not only inflammation but also fibroproliferative processes in ALI/ARDS as well as the development of IPF.

\section{SUPPORT STATEMENT}

This study was partly supported by a grant to the Diffuse Lung Diseases Research Group from the Ministry of Health, Labour and Welfare, Japan.

\section{STATEMENT OF INTEREST}

None declared.

\section{REFERENCES}

1 Chapman HA. Disorders of lung matrix remodeling. J Clin Invest 2004; 113: 148-157.

2 Phua J, Badia JR, Adhikari NK, et al. Has mortality from acute respiratory distress syndrome decreased over time? A systematic review. Am J Respir Crit Care Med 2009; 179: 220-227.

3 Marshall RP, Bellingan G, Webb S, et al. Fibroproliferation occurs early in the acute respiratory distress syndrome and impacts on outcome. Am J Respir Crit Care Med 2000; 162: 1783-1788.

4 Ichikado K, Suga M, Muranaka H, et al. Prediction of prognosis for acute respiratory distress syndrome with thin-section CT: validation in 44 cases. Radiology 2006; 238: 321-329.

5 Kasai H, Allen JT, Mason RM, et al. TGF- $\beta 1$ induces human alveolar epithelial to mesenchymal cell transition (EMT). Respir Res 2005; 6: 56.

6 Fahy RJ, Lichtenberger F, McKeegan CB, et al. The acute respiratory distress syndrome: a role for transforming growth factor- $\beta 1$. Am J Respir Cell Mol Biol 2003; 28: 499-503.

7 Chesnutt AN, Matthay MA, Tibayan FA, et al. Early detection of type III procollagen peptide in acute lung injury. Pathogenetic and prognostic significance. Am J Respir Crit Care Med 1997; 156: 840-845.

8 Gasse P, Mary C, Guenon I, et al. IL-1R1/MyD88 signaling and the inflammasome are essential in pulmonary inflammation and fibrosis in mice. J Clin Invest 2007; 117: 3786-3799.

9 Murray LA, Hackett TL, Warner SM, et al. BMP-7 does not protect against bleomycin-induced lung or skin fibrosis. PLoS One 2008; 3: e4039.

10 Scotton CJ, Krupiczojc MA, Konigshoff M, et al. Increased local expression of coagulation factor $\mathrm{X}$ contributes to the fibrotic response in human and murine lung injury. J Clin Invest 2009; 119: 2550-2563.

11 Scotton TH, Lee YH, Kim KH, et al. Role of lung apolipoprotein A-I in idiopathic pulmonary fibrosis: antiinflammatory and 
antifibrotic effect on experimental lung injury and fibrosis. Am J Respir Crit Care Med 2010; 182: 633-642.

12 Kawabata K, Hagio T, Matsumoto S, et al. Delayed neutrophil elastase inhibition prevents subsequent progression of acute lung injury induced by endotoxin inhalation in hamsters. Am J Respir Crit Care Med 2000; 161: 2013-2018.

13 Taooka Y, Maeda A, Hiyama K, et al. Effects of neutrophil elastase inhibitor on bleomycin-induced pulmonary fibrosis in mice. Am J Respir Crit Care Med 1997; 156: 260-265.

14 Suzuki T, Moraes TJ, Vachon E, et al. Proteinase-activated receptor-1 mediates elastase-induced apoptosis of human lung epithelial cells. Am J Respir Cell Mol Biol 2005; 33: 231-247.

15 Chambers RC. Procoagulant signalling mechanisms in lung inflammation and fibrosis: novel opportunities for pharmacological intervention? Br J Pharmacol 2008; 153: Suppl. 1, S367-S378.

16 Yamanouchi H, Fujita J, Hojo S, et al. Neutrophil elastase: $\alpha-1$ proteinase inhibitor complex in serum and bronchoalveolar lavage fluid in patients with pulmonary fibrosis. Eur Respir J1998; 11: 120-125.

17 Obayashi Y, Yamadori I, Fujita J, et al. The role of neutrophils in the pathogenesis of idiopathic pulmonary fibrosis. Chest 1997; 112: 1338-1343.

18 Mitsuhashi H, Asano S, Nonaka $\mathrm{T}$, et al. Administration of truncated secretory leukoprotease inhibitor ameliorates bleomycin-induced pulmonary fibrosis in hamsters. Am J Respir Crit Care Med 1996; 153: 369-374.

19 Chua F, Dunsmore SE, Clingen PH, et al. Mice lacking neutrophil elastase are resistant to bleomycin-induced pulmonary fibrosis. Am J Pathol 2007; 170: 65-74.
20 Ishii Y, Fujimoto S, Fukuda T. Gefitinib prevents bleomycininduced lung fibrosis in mice. Am J Respir Crit Care Med 2006; 174: 550-556.

21 Oga $T$, Matsuoka $T$, Yao $C$, et al. Prostaglandin $\mathrm{F}(2 \alpha)$ receptor signaling facilitates bleomycin-induced pulmonary fibrosis independently of transforming growth factor- $\beta$. Nat Med 2009; 15: 1426-1430.

22 Sisson TH, Mendez M, Choi K, et al. Targeted injury of type II alveolar epithelial cells induces pulmonary fibrosis. Am J Respir Crit Care Med 2010; 181: 254-263.

23 Kessenbrock K, Frohlich L, Sixt M, et al. Proteinase 3 and neutrophil elastase enhance inflammation in mice by inactivating antiinflammatory progranulin. J Clin Invest 2008; 118: 2438-2447.

24 Ask K. Targeting genes for treatment in idiopathic pulmonary fibrosis: challenges and opportunities, promises and pitfalls. Proc Am Thorac Soc 2006; 3: 389-393.

25 Cutroneo KR, White SL, Phan SH, et al. Therapies for bleomycin induced lung fibrosis through regulation of TGF- $\beta 1$ induced collagen gene expression. J Cell Physiol 2007; 211: 585-589.

26 Denholm EM, Rollins SM. Expression and secretion of transforming growth factor- $\beta$ by bleomycin-stimulated rat alveolar macrophages. Am J Physiol 1993; 264: L36-L42.

27 Nasreen N, Mohammed KA, Mubarak KK, et al. Pleural mesothelial cell transformation into myofibroblasts and haptotactic migration in response to TGF- $\beta 1$ in vitro. Am J Physiol Lung Cell Mol Physiol 2009; 297: L115-L124.

28 Brinkmann V, Reichard U, Goosmann C, et al. Neutrophil extracellular traps kill bacteria. Science 2004; 303: 1532-1535. 